UDC 511.6

\title{
A Definition of Type Domain of a Parallelotope
}

\author{
Grishukhin V. P. \\ Central Economics and Mathematics Institute RAS \\ Nakhimovskii prosp., 47, Moscow, 117418, Russia \\ e-mail: grishuhn@cemi.rssi.ru \\ received October 10, 2013
}

Keywords: parallelotope, type domain, zonotope

\begin{abstract}
Each convex polytope $P=P(\alpha)$ can be described by a set of linear inequalities determined by vectors $p$ and right hand sides $\alpha(p)$. For a fixed set of vectors $p$, a type domain $\mathcal{D}\left(P_{0}\right)$ of a polytope $P_{0}$ and, in particular, of a parallelotope $P_{0}$ is defined as a set of parameters $\alpha(p)$ such that polytopes $P(\alpha)$ have the same combinatorial type as $P_{0}$ for all $\alpha \in \mathcal{D}\left(P_{0}\right)$.

In the second part of the paper, a facet description of zonotopes and zonotopal parallelotopes are given.

The article is published in the author's wording.
\end{abstract}

\section{Properties of type domains}

A type of a polytope $P$, in particular, of a parallelotope, is an isomorphism class of the partial ordered set of all faces of $P$.

There corresponds a Voronoi polytope $P_{V}(f)$ to each positive semidefinite quadratic form $f$. Voronoi defined in his famous paper [1] an L-type domain of a Voronoi polytope $P_{V}\left(f_{0}\right)$ as a set of quadratic forms $f$ such that polytopes $P_{V}(f)$ have the same type as $P_{V}\left(f_{0}\right)$. Voronoi conjectured in [1] that each parallelotope is affinely equivalent to a Voronoi polytope. Since the Voronoi conjecture is not yet proved, it is useful to define a type domain of a parallelotope not using quadratic forms.

Call a face of codimension 1 by a facet. Each $n$-dimensional convex centrally symmetric polytope $P$ can be described by the following system of inequalities

$$
P=P(\alpha)=\left\{x \in \mathbb{R}^{n}:\langle p, x\rangle \leq \alpha(p), p \in \mathcal{P}\right\},
$$

where $\mathcal{P} \subset \mathcal{R}^{n}$ is a set of vectors including all facet vectors such that if $p \in \mathcal{P}$, then $-p \in \mathcal{P}$. Here $\langle p, x\rangle$ is scalar product of vectors $p, x \in \mathbb{R}^{n}$. The function $\alpha \in \mathbb{R}_{+}^{\mathcal{P}}$ is symmetric and non-negative, i.e. $\alpha(-p)=\alpha(p) \geq 0$ for all $p \in \mathcal{P}$. Call the function $\alpha$ by 
parameter. Let $\mathcal{P}(P) \subseteq \mathcal{P}$ be a set of all facet vectors of $P$. Suppose that, for any $p \in \mathcal{P}$, the following hyperplane

$$
H(\alpha, p)=\left\{x \in \mathbb{R}^{n}:\langle p, x\rangle=\alpha(p)\right\}
$$

supports $P$ at a face $G$, i.e. $H(\alpha, p) \cap P=G$. The face $G$ is a facet if $p \in \mathcal{P}(P)$.

Actually, I consider a family of polytopes $P(\alpha)$ for distinct $\alpha$, but for a fixed set of vectors $\mathcal{P}$. Each of polytopes of the family is obtained from any other by parallel shifts of supporting hyperplanes $H(\alpha, p)$.

A type domain $\mathcal{D}(P)$ of a polytope $P$ is a set of all parameters $\alpha$ such that polytopes $P(\alpha)$ have the same type as $P$ for all $\alpha \in \mathcal{D}(P)$. The domain $\mathcal{D}(P)$ is determined by equalities and inequalities between values $\alpha(p)$ for distinct $p$.

Let $P=P(\alpha)$ be a polytope described by (1). Let $\mathcal{P}(G) \subseteq \mathcal{P}(P)$ be a set of facet vectors of all facet containing $G$. The following assertion describes some equalities between parameters $\alpha(p)$.

Proposition 1. Let $G$ be a $k$-dimensional face of a polytope $P$. Then

(i) if $t \in \mathcal{P}-\mathcal{P}(G)$ is such that the hyperplane $H(\alpha, t)$ supports $P$ at the face $G$, then

$$
\alpha(t)=\sum_{p \in \mathcal{P}(G)} \mu_{t}(p) \alpha(p),
$$

where $\mu_{t}(p) \geq 0$ are coefficients of the decomposition $t=\sum_{p \in \mathcal{P}(G)} \mu_{t}(p) p$ of the vector $t$ by the facet vectors $p \in \mathcal{P}(G)$;

(ii) if $|\mathcal{P}(G)|>n-k$, then

$$
\sum_{p \in \mathcal{P}(G)} \mu(p) \alpha(p)=0
$$

where $\mu(p)$ are coefficients of a linear dependence $\sum_{p \in \mathcal{P}(G)} \mu(p) p=0$ between vectors $p \in \mathcal{P}(G)$.

Proof. (i) Since the hyperplane $H(\alpha, t)$ contains the face $G$, the vector $t$ lies in the space $X(G)$ that is orthogonal to affine space of the face $G$. The space $X(G)$ is generated by facet vectors $p \in \mathcal{P}(G)$. Moreover the vectors $p \in \mathcal{P}(G)$ generate a cone, where the vector $t$ lies. Therefore the following representation $t=\sum_{p \in \mathcal{P}(G)} \mu_{t}(p) p$ holds, where $\mu_{t}(p) \geq 0$ for all $p \in \mathcal{P}(G)$. Any point $x \in G$ satisfies the equality $\langle t, x\rangle=\alpha(t)$ and $\langle p, x\rangle=\alpha(p)$ for all $p \in \mathcal{P}(G)$. Multiplying the last equalities for $p \in \mathcal{P}(G)$ by $\mu_{t}(p)$ and summing over all $p$, we obtain the wanted representation of $\alpha(t)$ through parameters $\alpha(p)$.

(ii) Since the number of vectors $p \in \mathcal{P}(G)$ is greater than dimension of the space $X(G)$ generated by $p \in \mathcal{P}(G)$, there is a linear dependence $\sum_{p \in \mathcal{P}(G)} \mu(p) p=0$. As in the case (i), multiplying this dependence by $x \in G$, we obtain the wanted equality $\sum_{p \in \mathcal{P}(G)} \mu(p) \alpha(p)=0$.

If facets of a polytope are centrally symmetric, then they are organized in $k$-belts. Each $k$-belt of an $n$-polytope $P$ is uniquely determined by a family of mutually parallel $(n-2)$ faces that are intersections of neighboring facets of this $k$-belt. The following assertion describes linear inequalities between $\alpha(p)$ related to 6-belts of a polytope. 
Proposition 2. Let $p_{1}, p_{2}, p_{3}$ be facet vectors of a 6 -belt of a polytope $P$ such that $p_{3}=$ $\mu_{1} p_{1}+\mu_{2} p_{2}$, where $\mu_{1}, \mu_{2}>0$. Then this 6 -belt determines the following inequalities between the three parameters $\alpha\left(p_{i}\right), i=1,2,3$,

$$
\mu_{1} \alpha\left(p_{1}\right)+\mu_{2} \alpha\left(p_{2}\right) \geq \alpha\left(p_{3}\right) .
$$

Proof. The facet vectors $p_{1}, p_{2}, p_{3}$ of a 6 -belt of $P$ lie in a 2 -plane $\Pi_{2}$ that is orthogonal to mutually parallel $(n-2)$-faces of this belt. Therefore these three vectors are lnearly dependent. Let this dependence be $p_{3}=\mu_{1} p_{1}+\mu_{2} p_{2}$, where $\mu_{1}, \mu_{2}>0$. Any point $x \in H\left(\alpha, p_{1}\right) \cap H\left(\alpha, p_{2}\right)$ of the intersection of supporting hyperplanes of facets $F\left(p_{1}\right)$ and $F\left(p_{2}\right)$ is cut off from $P(\alpha)$ by the hyperplane $H\left(\alpha, p_{3}\right)$ that supports the facet $F\left(p_{3}\right)$. Hence, for this $x$, we have $\left\langle p_{3}, x\right\rangle \geq \alpha\left(p_{3}\right)$. Since $p_{3}=\mu_{1} p_{1}+\mu_{2} p_{2}$, this equality takes the form $\mu_{1}\left\langle p_{1}, x\right\rangle+\mu_{2}\left\langle p_{2}, x\right\rangle \geq \alpha\left(p_{3}\right)$. Obviously, the equalities $\left\langle p_{1}, x\right\rangle=\alpha\left(p_{1}\right)$ and $\left\langle p_{2}, x\right\rangle=\alpha\left(p_{2}\right)$ hold. These two equalities and the above inequality give the triangle inequality (3).

Since the 3 facet vectors $p_{i}$ for $i=1,2,3$ are equivalent, each 6 -belt gives 3 inequalities of type (3). If at least one of these inequalities, say (3), holds as equality, then the 6-belt is transformed into a 4 -belt $\left(p_{1}, p_{2}\right)$. In this case $p_{3}$ is not a facet vector, i.e. $p_{3} \notin \mathcal{P}(P)$.

\section{Parallelotopes}

Proposition 1 gives nothing for a primitive parallelotope. If $P(\alpha)$ is a primitive or zonotopal parallelotope, then one can choose length of facet vectors $p$ such that $\alpha(p)=$ $\langle p, D p\rangle$ for all $p \in \mathcal{P}$, where $D$ is a positive definite matrix, and the type domain $\mathcal{D}(P)$ is determined by matrices $D$ (see [1] and [6]).

If $P(\alpha)$ is a parallelotope, then each facet of it is centrally symmetric. Facets are special cases of standard faces of a parallelotope. Standard faces were defined by Dolbilin in [5]. Each standard face $F$ is centrally symmetric. It is useful consider parallelotopes described by (1), where hyperplanes $H(\alpha, p)$ for all $p \in \mathcal{P}$ support standard faces of $P(\alpha)$. Vectors $p$ of these hyperplanes may be find using item (i) of Proposition 1.

Let $c_{p}$ be the center of a standard face $F(p)$ determined by a vector $p \in \mathcal{P}$. Then the parallelotope $P(\alpha)$ can be described by the inequalities (1) with $\alpha(p)=\left\langle p, c_{p}\right\rangle$.

Theorem 1. A parallelotope $P(\alpha)$ is affinely equivalent to a Voronoi polytope if and only if there are lengths of facet vectors $p$ such that $\alpha(p)=\langle p, D p\rangle$ for some positive definite matrix $D$.

Proof. Let $P(\alpha)$ be a parallelotope that is affinely equivalent to a Voronoi polytope. It is proved in [4] that then one can choose lengths of facet vectors $p$ such that $c_{p}=D p$, where $D$ is a positive semi-definite matrix.

Conversely, if $\alpha(p)=\langle p, D p\rangle$, then $c_{p}=D p$. By [4], this means that $P(\alpha)$ is affinely equivalent to a Voronoi polytope.

If $P=P(\alpha)$ is not primitive, then $P$ has $k$-faces $G$ such that $|\mathcal{P}(G)|>n-k$. In this case, the type domain $\mathcal{D}(P)$ is a face of the type domain of a primitive parallelotope.

Call a parallelotope $P$ rigid if its type domain $\mathcal{D}(P)$ is one-dimensional. For a rigid parallelotope $P$, Proposition 1 allows to prove its rigidity, since a rigid parallelotope has sufficiently many $k$-faces $G$ with $|\mathcal{P}(G)|>n-k$. In particular, one can show by this method that the Voronoi polytopes $P_{V}\left(D_{4}\right), P_{V}\left(E_{n}\right), P_{V}\left(E_{n}^{*}\right)$ for $n=6,7$, are rigid. 


\section{Zonotopes}

In this section I give explicit expressions of $\alpha(p)$ for zonotopes and zonotopal parallelotopes.

Recall that a zonotope $Z(U)=\sum_{u \in U} b_{u} z(u)$ generated by a set of vectors $U \subset \mathbb{R}^{n}$ is the Minkowski sum of weighted segments

$$
z(u)=\left\{x \in \mathbb{R}^{n}: x=\lambda u:-1 \leq \lambda \leq 1\right\} .
$$

Hence

$$
Z(U)=\left\{x \in \mathbb{R}^{n}: x=\sum_{u \in U} \lambda_{u} u: \quad-b_{u} \leq \lambda_{u} \leq b_{u}, u \in U\right\} .
$$

Here $b_{u} \geq 0$ are non-negative weights for all $u \in U$. Below, $Z(U)$ denotes always the above sum. This is in a sense a "vertex description"of the zonotope $Z(U)$. Each vertex $v$ of $Z(U)$ has a description $v=\sum_{u \in U} b_{u}( \pm u)$, where from two signs \pm only one is taken. But we need a facet description of $Z(U)$.

Let $p \in \mathbb{R}^{n}$ be a vector. Define a subset $U_{p} \subseteq U$ as follows.

$$
U_{p}=\{u \in U:\langle p, u\rangle=0\} .
$$

The following Lemma 1 helps to find a facet description of $Z(U)$.

Lemma 1. A shift of the zonotope $Z\left(U_{p}\right)$ is a face $G$ of the zonotope $Z(U)$. The center of the face $G$ is an end-point of the vector

$$
c_{p}=\sum_{u \in U-U_{p}} b_{u} \frac{\langle p, u\rangle}{|\langle p, u\rangle|} u .
$$

The affine hyperplane

$$
H_{p}=\left\{x \in \mathbb{R}^{n}:\langle p, x\rangle=\left\langle p, c_{p}\right\rangle\right\}
$$

supports $Z(U)$ at the face $G=c_{p}+Z\left(U_{p}\right) \subset H_{p}$. In particular, if $U_{p}=U$, then $c_{p}=0$ and $G=Z(U)$, and if $U_{p}=\emptyset$, then $G$ is a vertex that coincides with $c_{p}$.

Proof. Using (4), it is easy to see that $c_{p}$ is a point of $Z(U)$. We show that the affine hyperplane $H_{p}$ supports $Z(U)$, i.e. $\langle p, x\rangle \leq\left\langle p, c_{p}\right\rangle$ for all $x \in Z(U)$. It is sufficient to verify these inequalities for vertices. We have $\langle p, v\rangle=\sum_{u \in U} b_{u}( \pm\langle p, u\rangle)$. Since $b_{u} \geq 0$ and $\langle p, u\rangle \in\{0, \pm 1\}|\langle p, u\rangle|$ for all $u \in U$, the following inequality holds

$$
\langle p, v\rangle \leq \sum_{u \in U-U_{p}} b_{u} \frac{(\langle p, u\rangle)^{2}}{|\langle p, u\rangle|}=\left\langle p, c_{p}\right\rangle .
$$

This inequality implies that the hyperplane $H_{p}$ supports $Z(U)$, and $c_{p}+Z\left(U_{p}\right)=G$ is a face of $Z(U)$.

Lemma 1 implies the following important

Theorem 2. Let $U \subset \mathbb{R}^{n}$ be a set of vectors. Let $b_{u} \geq 0$ be non-negative weights for all $u \in U$. Let $\mathcal{P}$ be a set of vectors containing all facet vectors $p$ of the zonotope $Z(U)=\sum_{u \in U} b_{u} z(u)$. Then the zonotope $Z(U)$ has the following description by linear inequalities

$$
Z(U)=\left\{x \in \mathbb{R}^{n}:\langle p, x\rangle \leq \alpha_{U}(p) \text { for all } p \in \mathcal{P}\right\}
$$


where

$$
\alpha_{U}(p)=\sum_{u \in U-U_{p}} b_{u} \frac{(\langle p, u\rangle)^{2}}{|\langle p, u\rangle|} .
$$

Each inequality in (7) supports a face of $Z(U)$.

Consider a zonotope $Z(U)$ generated by a unimodular set of vectors $U$. It is known (see, for example, [7], [3]) that a zonotope $Z(U)$ is a parallelotope if and only if vectors $u \in U$ are in proportion with vectors of a unimodular set of vectors. According to description of $Z(U)$ by (4), we can suppose that the set $U$ is itself unimodular. Recall that a set is unimodular if each its vector has an integral representation in any its basic subset.

Let $F$ be a facet of $Z(U)$ with its facet vector $p$ which is, recall, orthogonal to $F$. The facet $F$ is also a zonotope $Z\left(U_{F}\right)$, where $U_{F}=U_{p}$. The unimodular set $U$ represents a regular matroid $M_{U}$ (see any book on Matroid Theory, for example, [2]). The subset $U_{F}$ represents a copoint of $M_{U}$. A definition of a copoint implies that the set of equalities $\langle p, u\rangle=0$ for all $u \in U_{F}$ determines uniquely up to a multiple a facet vector $p$. It is known, see, for example, [2], [3], that, for any copoint $U_{F}$ of a regular matroid, lengths of its facet vectors $p$ can be chosen such that they satisfy the following condition

$$
\langle p, u\rangle \in\{0, \pm 1\} \text { for all } u \in U .
$$

It is important that fulfillment of this condition for all facet vectors is equivalent to unimodularity of the set $U$ (see, for example, [2], [3]).

Let a vector $p$ satisfies the condition (9). Then $|\langle p, u\rangle| \in\{0,1\}$, and, since $\langle p, u\rangle=0$ for $u \in U_{p}, c_{p}$ can be written as

$$
c_{p}=\sum_{u \in U} b_{u}(\langle p, u\rangle) u
$$

Theorem 3. Let $U \subset \mathbb{R}^{n}$ be a unimodular set of vectors. Let $b_{u} \geq 0$ be non-negative weights for all $u \in U$. Let $\mathcal{P}$ be a set of all facet vectors $p$ of the zonotope $Z(U)=$ $\sum_{u \in U} b_{u} z(u)$. Let facet vectors are chosen such that they satisfy conditions (9). Then the zonotope $Z(U)$ has the following description by linear inequalities

$$
Z(U)=\left\{x \in \mathbb{R}^{n}:\langle p, x\rangle \leq f_{U}(p) \text { for all } p \in \mathcal{P}\right\},
$$

where

$$
f_{U}(p)=\sum_{u \in U} b_{u}(\langle p, u\rangle)^{2}=\left\langle p, \sum_{u \in U} b_{u}\left(u u^{T}\right) p\right\rangle,
$$

is a positive semi-definite quadratic form on vectors $p \in \mathcal{P}$.

Proof. If $p$ is a facet vector, then it satisfies conditions (9). Hence $|\langle p, u\rangle|=1$ for all $u \in U-U_{p}$ and the hyperplane (6) takes the form

$$
H_{p}(f)=\left\{x \in \mathbb{R}^{n}:\langle p, x\rangle=f_{U}(p)\right\} .
$$

This implies the assertion of this theorem.

By Theorem 1, Theorem 3 shows that the zonotopal parallelotope $Z(U)$ is affinely equivalent to a Voronoi polytope. 


\title{
References
}

1. G.F. Voronoi, Nouvelles applications de paramètres continus á la théorie de forms quadratiques, Deuxième memoire, J. reine angew. Math. 134 (1908), 198-287, 136 (1909), $67-178$.

2. M. Aigner, Combinatorial Theory, Springer-Verlag, 1979.

3. M. Deza, V. Grishukhin, Voronoi's conjecture and space tiling zonotopes, Mathematika $\mathbf{5 1}$ (2004) 1-10.

4. M. Deza, V. Grishukhin, Properties of parallelotopes equivalent to Voronoi's conjecture, Europ. J. Combinatorics 25 (2004) 517-533.

5. N.P. Dolbilin, Properties of faces of parallelohedra, Proc. Steklov Inst. of Math. 266 (2009) $112-126$.

6. R.M. Erdahl, Zonotopes, Dicings, and Voronoi's conjecture on Parallelohedra, Eur. J. Combin. 20 (1999) 527-549.

7. A. Björner, M. Las Vergnas, B. Sturmfels, N. White, G.H. Ziegler, Oriented Matroids, (Encyclopedia of Mathematics and its Applications 46) Cambridge Univ. Press (1999).

\section{Определение области типа параллелоэдра}

\author{
Гришухин В.П. \\ Центральный экономико-математический институт РАН \\ 117418 Россия, г. Москва, Нахимовский просп., 47
}

Ключевые слова: параллелоэдр, область типа, зонотоп

Любой выпуклый многогранник $P=P(\alpha)$ может быть описан системой линейных неравенств, определяемых векторами $p$ и правыми частями $\alpha(p)$. Для фиксированного множества векторов $p$ определяется область типа $\mathcal{D}\left(P_{0}\right)$ многогранника $P_{0}$, и в частности параллелоэдра $P_{0}$, как такое множество параметров $\alpha(p)$, что многогранники $P(\alpha)$ имеют тот же комбинаторный тип, что и $P_{0}$ для всех $\alpha \in \mathcal{D}\left(P_{0}\right)$. Во второй части статьи дается фасетное описание зонотопов и зонотопных параллелоэдров.

Статья публикуется в авторской редакции.

\section{Сведения об авторе: \\ Гришухин Вячеслав Петрович,}

Центральный экономико-математический институт РАН

д-р физ.-мат. наук 